\title{
Complete metabolic response of metastatic castration-resistant neuroendocrine carcinoma of the prostate after treatment with RRx-001 and reintroduced platinum doublets
}

\author{
Michelle Ojemuyiwa $^{1}$ | Karen Zeman $^{1}$ | Alexander Spira ${ }^{2}$ | Bryan Oronsky ${ }^{3}$ (D) |

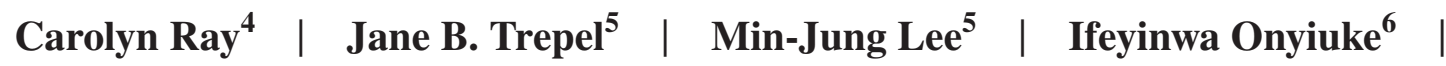 \\ Christina Brzezniak ${ }^{1}$
}

${ }^{1}$ Walter Reed National Military Medical Center, Bethesda, Maryland

${ }^{2}$ Virginia Cancer Specialists (VCS), Fairfax, Virginia

${ }^{3}$ EpicentRx, San Diego, California

${ }^{4}$ St. Francis Hospital \& Medical Center, Hartford, Connecticut

${ }^{5}$ Developmental Therapeutics

Branch, National Cancer Institute, NIH,

Bethesda, Maryland

${ }^{6}$ VA Connecticut Health Care System, West Haven, Connecticut

Correspondence

Bryan Oronsky, EpicentRx, San Diego, CA.

Email: info@epicentrx.com

\section{Key Clinical Message}

Presented herein is the case of a heavily pretreated patient with high-grade neuroendocrine prostate cancer that achieved a complete metabolic response on platinumbased chemotherapy after treatment with the dual CD-47 and SIRP- $\alpha$ inhibitor, RRx-001, in a Phase II clinical trial.

\section{K E Y W O R D S}

macrophage repolarization, neuroendocrine cancer, platinum doublets, priming, prostate cancer, RRx-001

\section{1 | INTRODUCTION}

Prostate cancer (PC) is the most common malignancy in US men (excluding nonmelanoma skin cancer) and the second leading cause of death after non-small-cell lung cancer (NSCLC). ${ }^{1}$ The mainstays of treatment for metastatic and locally advanced prostate cancer are androgen deprivation therapies (ADT), such as bicalutamide and/or leuprolide. These ultimately fail due to castrate resistance, which may be treated with sequential use of abiraterone, enzalutamide and followed by docetaxel, cabazitaxel, or sipuleucel-T. Regretfully, all of these therapies eventually and inevitably engender resistance, which is uniformly fatal, due to the development and proliferation of androgen-resistant cells. ${ }^{2}$ The neuroendocrine (NE) cell, initially a minor and widely interspersed cell population in normal human prostate glands, may proportionally increase with the progression to the advanced, hormone-resistant state ${ }^{3}$ following long-term androgen deprivation therapy (Figure 1). Since NE cells are negative for prostate-specific antigen (PSA) and androgen receptor (AR), progression of ADT-treated prostate cancer in the setting of negative or low levels of PSA suggests the possibility of neuroendocrine transformation.

Consistent with the hypothesis that ADT is responsible for or induces NE conversion, (a) overtde novo disease is extremely rare $(<0.1 \%$ of all diagnosed $\mathrm{PCs}),{ }^{4}$ (b) a higher proliferation index of tumor cells that are located in close proximity to the NE cell foci has been observed, and (c) the secretory products of NE cells have been shown to inhibit apoptosis, ${ }^{5}$ suggesting that the neuroendocrine cells actively aid and abet carcinogenesis. ${ }^{6}$

Treatment-emergent or secondary neuroendocrine prostate cancer (t-NEPC) is thought to occur in up to $25 \%$ of 
Androgen-dependent cancer

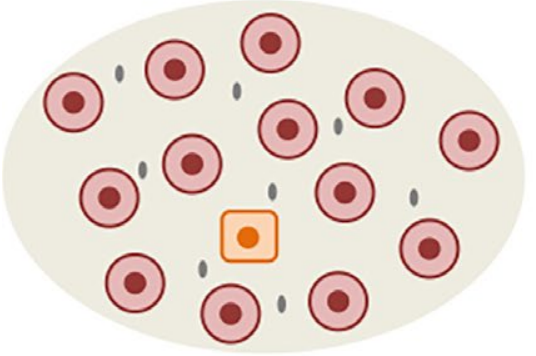

Androgen-deprived cancer

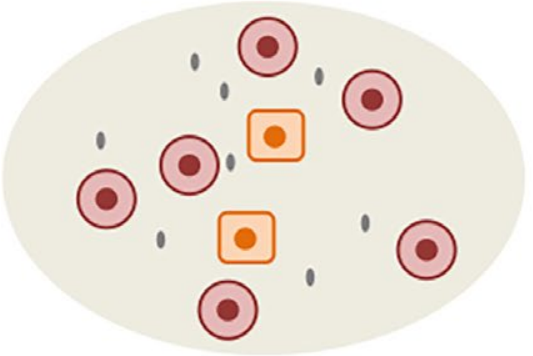

Androgen-independent cancer

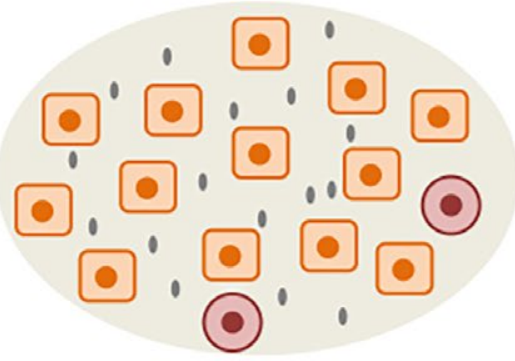

Epithelial cell Neuroendocrine cell

F I G U R E 1 In normal prostatic parenchyma, the neuroendocrine (NE) cells are rare and widely interspersed. Neuroendocrine cells may "take over" after androgen deprivation therapy (ADT) eliminates the androgen-sensitive cells

patients with advanced PC. ${ }^{7,8}$ Prostate-specific antigen (PSA) is an unreliable marker of disease activity since neuroendocrine cells do not express it, contributing to late-stage recognition of t-NEPC during ADT and its overall poor prognosis. Symptoms are generally related to obstruction, with episodes of urinary retention and hematuria. ${ }^{9}$ Based on the treatment paradigm for small-cell lung cancer, as the prototype of aggressive neuroendocrine tumors, patients are often treated with platinum-based chemotherapy and radiation as appropriate. However, despite initial responses, rapid progression is the rule and no standard second line or curative therapy is available. ${ }^{10}$ This report presents a case of a complete metabolic regression of t-NEPC after sequential treatment with the experimental macrophage M1 repolarizing agent, RRx-001, dosed intravenously once weekly until progression, followed by reintroduced carboplatin and etoposide on a multi-arm, multi-center clinical trial called QUADRUPLE THREAT (NCT02489903).

\section{2 | CASE PRESENTATION}

The subject of this case report is a 65-year-old male diagnosed with pT3a, N0, Mx, Gleason score $9(4+5)$ adenocarcinoma of the prostate diagnosed in 2008, for which he received radical retropubic prostatectomy (RRP) followed by external beam radiation therapy (EBRT). Due to a prostate-specific antigen doubling time of $<3$ months in 2012, he was started on combined upfront androgen deprivation therapy with bicalutamide and goserelin. Subsequent therapies included enzalutamide plus an experimental prostate-specific antigen (PSA)-TRICOM viral vaccine (PROSTVAC), and docetaxel. Neuroendocrine differentiation was diagnosed by needle biopsy and the patient was treated with six cycles of carboplatin plus etoposide from April 2016 to September 2016.
On 09 November 2016, with progressive disease in the pelvis (enlargement of retroperitoneal lymphadenopathy), the patient enrolled on the Phase II QUADRUPLE THREAT clinical trial in which RRx-001 is dosed weekly intravenously until progression followed by rechallenge with platinum and etoposide.

The patient began weekly RRx-001 infusions on 23 November 2016. He received a second dose on 29 November and a third on 6 December 2016. On 7 December after three doses of RRx-001, the patient presented to the hospital with lower back pain. Magnetic resonance imaging (MRI) of the brain done at that time demonstrated lesions that had not been imaged previously (because baseline cranial imaging had not been required) and, the patient immediately received two weeks of whole brain radiation therapy.

The patient was reimaged with positron emission tomography/computed tomography (PET/CT) on 28 December 2016 and this scan showed RECIST evidence of progression in the previously documented retroperitoneal lymphadenopathy. There was significant enlargement and increased fluorodeoxyglucose (FDG) avidity of the retroperitoneal mass that was confluent with the left kidney, causing obstruction at the ureteropelvic junction. In addition, new liver lesions were discovered (Figure 2). RRx-001 was discontinued and the patient was transitioned back to carboplatin and etoposide rechallenge on 4 January 2017.

On 19 January, day 15 of his first cycle, the patient developed hematuria with urinary clotting, anemia (hemoglobin $7.1 \mathrm{~g} / \mathrm{dL}$ ), renal insufficiency (creatinine $1.42 \mathrm{mg} / \mathrm{dL}$ ), and symptoms of obstruction. Over the month of January, 2017, these symptoms recurred, requiring two separate hospitalizations. Treatment for the hematuria, clotting, and anemia involved continuous bladder irrigation and red blood cell transfusion. After two cycles of platinum doublets, reimaging demonstrated a complete metabolic response on qualitative 

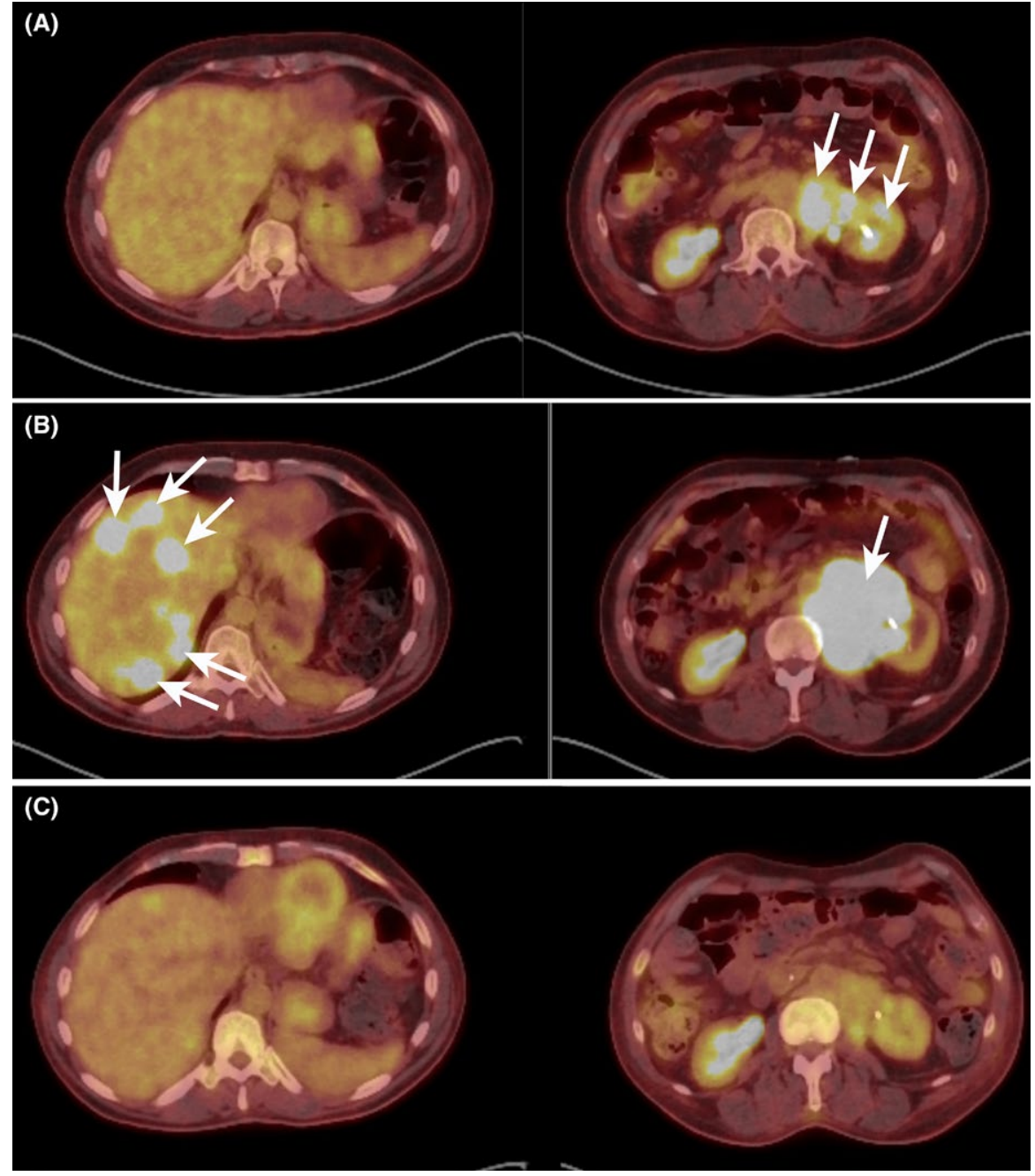

F IG URE 2 A, Axial fused positron emission tomography/computed tomography (PET/CT) enrollment scan: liver on left shows diffuse background parenchymal activity with no discrete lesions. Image on the right shows large, highly fluorodeoxyglucose (FDG) avid nodal conglomerate at left ureteropelvic junction. B, Images after three doses of RRx001: Multiple discrete, highly FDG avid lesions in both the left and right lobes of the liver with significant increase in size and FDG avidity of retroperitoneal nodal mass. Note the mass effect on the kidney. C, Scan after two cycles of platinum doublets: extensive regression of liver lesions and complete metabolic response with activity approximating tissue background levels. Significant decrease in size with a complete metabolic response in retroperitoneal nodal mass evaluation of FDG PET, even though his tumors were morphologically stable.

In addition, the hematuria and clotting subsided, the patient's quality of life drastically improved and the creatinine decreased from 1.42 to $1.2 \mathrm{mg} / \mathrm{dL}$.

\section{3}

\section{DISCUSSION}

An under-recognized diagnosis, treatment-emergent t-NEPC, which may result from androgen deprivation therapy, due to the preferential selection and outgrowth of NE clones, is an incurable and highly lethal disease subtype with no available standard options in the second line after treatment with platinum doublets. High rates of initial responses to platinum doublets are followed inevitably by the development of intractable resistance. $\mathrm{RRx}-001$, a minimally toxic macrophage repolarizing agent with epigenetic properties, has been demonstrated to resensitize to previously tried and failed standard chemotherapies. $^{11}$

In the Phase II QUADRUPLE THREAT trial, RRx-001 is administered as a pretreatment or primer until progression in patients with four tumor types, SCLC, ovarian cancer, neuroendocrine, andepidermal growth factor receptor (EGFR) mutated NSCLC, prior to rechallenge with platinum doublets. RRx-001 is an epigenetic inhibitor and dual checkpoint inhibitor of CD47-SIRP $\alpha$ inhibition, which repolarizes M2like tumor-associated macrophages to M1-like macrophages. RRx-001, which has been associated with pseudoprogres$\operatorname{sion}^{12}$ due to biopsy-proven immune cell infiltration, ${ }^{13}$ edema, necrosis, and inflammation, appears to have elicited a similar pseudoprogressive enlargement of the tumors of this patient, (although in the absence of a biopsy, differentiating between true progressive disease and pseudoprogression is impossible), which might have suggested that he was in the throes of a death spiral rather than on the cusp of a complete metabolic response with platinum doublets.

These cases serve as an object lesson that marked clinical deterioration and radiographic worsening do not necessarily equal treatment failure in RRx-001 patients, given the activity of RRx-001 as primer, which enhances responses to subsequent chemotherapies. ${ }^{14,15}$ Therefore, despite initial appearances of rapidly progressive disease after only three doses of RRx-001, which might otherwise and in another context 
have warranted a referral to hospice, the best decision for this patient was not to withdraw treatment but to continue it per protocol with reintroduced platinum doublets. In another patient on the QUADRUPLE THREAT trial with high-grade extrapulmonary neuroendocrine disease and a durable partial response to single agent RRx-001, documented elsewhere, ${ }^{16}$ an increase in the size of his tumors preceded significant regression. Serial biopsies of his lesions demonstrated an influx of $\mathrm{CD}^{+}$lymphocytes, which appeared after 12 weeks.

In summary, given the absence of secondary salvage treatment options in t-NEPC, it is hoped that resensitization to initially highly effective platinum doublets after "priming" with RRx-001 will provide significant clinical benefit, even if as in the case of this patient rapid clinical worsening preceded it, and markedly change the bleak prognosis of this disease.

\section{INFORMED CONSENT}

Human Subjects: Informed consent was obtained by all participants in this study.

\section{ACKNOWLEDGMENTS}

The authors gratefully acknowledge the invaluable assistance of Dr. Corey A. Carter in the writing of this case report.

\section{CONFLICT OF INTEREST}

None declared.

\section{AUTHOR CONTRIBUTION}

MO: treated the patient. MO, KZ, AS, BO, CR, JT, ML, IO, and $\mathrm{CB}$ : wrote and edited the manuscript.

\section{ORCID}

Bryan Oronsky (D) http://orcid.org/0000-0002-3940-6329

\section{REFERENCES}

1. Siegel R, Naishadham D, Jemal A. Cancer statistics 2012. CA Cancer J Clin. 2012;62:10-29.

2. Karantanos T, Corn PG, Thompson TC. Prostate cancer progression after androgen deprivation therapy: mechanisms of castrate resistance and novel therapeutic approaches. Oncogene. 2013;32(49):5501-5511.
3. Weinstein MH, Partin AW, Veltri RW, Epstein JI. Neuroendocrine differentiation in prostate cancer: enhanced prediction of progression after radical prostatectomy. Hum Pathol. 1996;27:683-687.

4. Humphrey PA. Histological variants of prostatic carcinoma and their significance. Histopathology. 2012;60:59-7410.

5. Abrahamsson PA. Neuroendocrine cells in tumour growth of the prostate. Endocr Relat Cancer. 1999;6:503-1910.

6. Bonkhoff H, Wernert N, Dhom G, Remberger K. Relation of endocrine-paracrine cells to cell proliferation in normal, hyperplastic, and neoplastic human prostate. Prostate. 1991;19:91-98.

7. Beltran H, Tagawa ST, Park K, et al. Challenges in recognizing treatment-related neuroendocrine prostate cancer. J Clin Oncol. 2012;30:e386-e910.

8. Lotan TL, Gupta NS, Wang W, et al. ERG gene rearrangements are common in prostatic small cell carcinomas. Mod Pathol. 2011;24:820-828.

9. Stein ME, Bernstein Z, Abacioglu U, et al. Small cell (neuroendocrine) carcinoma of the prostate: Etiology, diagnosis, prognosis, and therapeutic implications - A retrospective study of 30 patients from the rare cancer network. Am J Med Sci. 2008;336:478-488.

10. Terry S, Beltran H. The many faces of neuroendocrine differentiation in prostate cancer progression. Front Oncol. 2014;4:60.

11. Oronsky B, Paulmurugan R, Foygel K, et al. RRx-001: a systemically non-toxic M2-to-M1 macrophage stimulating and prosensitizing agent in Phase II clinical trials. Expert Opin Investig Drugs. 2017;26(1):109-119.

12. Reid T, Oronsky B, Scicinski J, et al. Safety and activity of RRx-001 in patients with advanced cancer: a first-in-human, open-label, dose-escalation phase 1 study. Lancet Oncol. 2015;16(9):1133-1142.

13. Zhao H, Ning S, Nolley R, et al. The immunomodulatory anticancer agent, RRx-001, induces an interferon 4 through epigenetic induction of viral mimicry. Clin Epigenetics. 2017;9:4.

14. Carter CA, Oronsky B, Caroen S, et al. Partial response in an RRx-001-primed patient with refractory small-cell lung cancer after a third introduction of platinum doublets. Case Rep Oncol. 2016;9(2):285-289.

15. Carter CA, Oronsky B, Caroen S, et al. Partial response to carboplatin in an RRx-001 pretreated patient with EGFR-inhibitorresistance and T790M-negative NSCLC. Respir Med Case Rep. 2016;18:62-65.

16. Carter CA, Schmitz B, Peterson PG, et al. Immune reactivity and pseudoprogression or tumor flare in a serially biopsied neuroendocrine patient treated with the epigenetic agent RRx-001. Case Rep Oncol. 2016;9(1):164-170.

How to cite this article: Ojemuyiwa M, Zeman K, Spira A, et al. Complete metabolic response of metastatic castration-resistant neuroendocrine carcinoma of the prostate after treatment with RRx-001 and reintroduced platinum doublets. Clin Case Rep. 2018;6:2478-2481. https://doi.org/10.1002/ $\underline{\mathrm{ccr} 3.1880}$ 